\title{
Common genetic variants do not associate with CAD in familial hypercholesterolemia
}

\author{
Erik PA van Iperen ${ }^{\star, 1,2,8}$, Suthesh Sivapalaratnam ${ }^{3,8}, \mathrm{~S}$ Matthijs Boekholdt ${ }^{3,4}$, G Kees Hovingh ${ }^{3}$, \\ Stephanie Maiwald ${ }^{3}$, Michael W Tanck ${ }^{1}$, Nicole Soranzo ${ }^{5}$, Jonathan C Stephens ${ }^{6,7}$, Jennifer G Sambrook ${ }^{6,7}$, \\ Marcel Levi ${ }^{3}$, Willem H Ouwehand ${ }^{5,6,7}$, John JP Kastelein ${ }^{3}$, Mieke D Trip ${ }^{4}$ and Aeilko H Zwinderman ${ }^{1}$
}

In recent years, multiple loci dispersed on the genome have been shown to be associated with coronary artery disease (CAD). We investigated whether these common genetic variants also hold value for CAD prediction in a large cohort of patients with familial hypercholesterolemia (FH). We genotyped a total of 41 single-nucleotide polymorphisms (SNPs) in 1701 FH patients, of whom 482 patients (28.3\%) had at least one coronary event during an average follow up of 66 years. The association of each SNP with event-free survival time was calculated with a Cox proportional hazard model. In the cardiovascular disease risk factor adjusted analysis, the most significant SNP was rs1122608:G > T in the SMARCA4 gene near the LDL-receptor (LDLR) gene, with a hazard ratio for CAD risk of $0.74(95 \% \mathrm{Cl} 0.49-0.99 ; P$-value 0.021$)$. However, none of the SNPs reached the Bonferroni threshold. Of all the known CAD loci analyzed, the SMARCA4 locus near the LDLR had the strongest negative association with CAD in this high-risk FH cohort. The effect is contrary to what was expected. None of the other loci showed association with CAD.

European Journal of Human Genetics (2014) 22, 809-813; doi:10.1038/ejhg.2013.242; published online 13 November 2013

Keywords: CAD; FH; GWAS; association

\section{INTRODUCTION}

Familial hypercholesterolemia $(\mathrm{FH})$ is an autosomal dominant disorder characterized by increased low-density lipoprotein cholesterol (LDL-C) levels and preponderance to coronary artery disease (CAD). The diagnosis is based on stringent clinical criteria or on the identification of mutations in the LDL-receptor (LDLR), apolipoprotein B or proprotein convertase subtilisin/kexin type 9 (PCSK9) gene. The frequency of heterozygosity is at least $1 / 500$ in most European countries. ${ }^{1}$ By virtue of the elevated LDL-C levels, FH results in lipid accumulation in the arterial wall and as a consequence accelerated atherosclerosis. $^{2,3}$ If left untreated, $50 \%$ of male and $30 \%$ of female heterozygous $\mathrm{FH}$ patients will develop CAD before 60 years of age. ${ }^{4}$ The age of onset and severity of CAD varies considerably between $\mathrm{FH}$ patients, even among individuals who share an identical gene defect. ${ }^{5}$

Previously, we performed a retrospective multicentre cohort study of $2400 \mathrm{FH}$ patients, of whom 782 patients $(32.6 \%)$ had at least one cardiovascular event during an average follow up of 66 years. ${ }^{6}$ In this cohort, we demonstrated that LDL-C levels are more important than the LDLR mutation type in determining the age of onset of CAD. ${ }^{7} \mathrm{We}$ also showed that classical risk factors including male gender, smoking, hypertension, type 2 diabetes, low levels of high-density lipoprotein cholesterol (HDL-C) and elevated lipoprotein(a) levels were independent risk factors for the development of CAD. However, these factors combined explained only $18.7 \%$ of the variation in CVD occurrence. $^{8}$ Thus, a considerable part of the variability in CVD occurrence remains to be disentangled and common genetic variation might provide one of the explanations.

Recent large-scale genome-wide association (GWA) studies have revealed common genetic variations at 45 loci that moderately affect (hazard ratios (HR) varying between 1.1 and 2.0) the incidence of CAD in the general Caucasian population. ${ }^{9-12}$

We set out to address whether common variations within the 45 previously identified loci by recent GWA studies are modifiers of $\mathrm{CAD}$ risk in a high-risk population of heterozygous $\mathrm{FH}$ cases.

\section{MATERIALS AND METHODS}

\section{Ethics statement}

Written informed consent was obtained from all patients. The Medical Ethics Review Board of each participating hospital approved the protocol, which complies with the Declaration of Helsinki.

\section{Study design and study population}

The Genetic Identification of Risk Factors in Familial Hypercholesterolemia (GIRaFH) is a retrospective multicenter cohort study. The study design and study population have been described elsewhere. ${ }^{6}$ Briefly, DNA samples from patient who, based on clinically oriented algorithms are anticipated to suffer from $\mathrm{FH}$ are being sent in to the central core molecular diagnostic laboratory by physicians working at one of the nationwide lipid clinics. LDLR gene variation was genotyped according to previously published methods. ${ }^{13}$ DNA of a total of 9300 hypercholesterolemic patients was stored in the DNA database at the time of initiation of the cohort. Only those cases from larger lipid clinics

\footnotetext{
${ }^{1}$ Department of Clinical Epidemiology, Biostatistics and Bioinformatics, Academic Medical Centre, Amsterdam, The Netherlands; ${ }^{2}$ Durrer Center for Cardiogenetic Research, Amsterdam, The Netherlands; ${ }^{3}$ Department of Vascular Medicine, Academic Medical Centre, Amsterdam, The Netherlands; ${ }^{4}$ Department of Cardiology, Academic Medical Centre, Amsterdam, The Netherlands; ${ }^{5}$ Human Genetics, Welcome Trust Sanger Institute, Hinxton, UK; ${ }^{6}$ Department of Haematology, University of Cambridge, Cambridge, UK; 7 National Health Service Blood and Transplant, Cambridge, UK

${ }^{8}$ These authors contributed equally to this work.

*Correspondence: EPA van Iperen, Department of Clinical Epidemiology, Biostatistics and Bioinformatics, Academic Medical Centre, J1B-207, Meibergdreef 9, 1105 AZ Amsterdam, The Netherlands. Tel: +312 0566 6959; Fax: +312 0566 9343; E-mail: e.p.vaniperen@amc.uva.nl
}

Received 11 February 2013; revised 6 September 2013; accepted 20 September 2013; published online 13 November 2013 
were selected (9188) for further analysis, as smaller clinics normally only send DNA samples of the rare, usually very serious FH cases. Of this set, 4000 cases were randomly selected. After review of medical records, a group of 2400 patients fulfilled the FH diagnostic criteria based on internationally established criteria and were included in the study. ${ }^{14}$ Phenotypic, CVD event and cause of death data were acquired from the medical charts. None of the study population received primary prevention in the form of beta-blockers or aspirin. $\mathrm{CAD}$ was defined as angina pectoris (AP), acute coronary syndrome (ACS), percutaneous coronary interventions (PCI) or coronary artery bypass grafting (CABG)

\section{SNP selection}

On the basis of the latest published meta-analysis, a total of 45 singlenucleotide polymorphisms (SNPs) associated with CAD were identified. ${ }^{12}$ We did not include SNPs which were only associated with an intermediate trait such as lipid levels, type 2 diabetes or hypertension.

If these SNPs were not directly genotyped, imputed data, using MACH and the HapMap phase 2 data sets (build 36 release 22), ${ }^{15-17}$ were used. Finally if this failed proxies were looked up within a window of $500 \mathrm{~kb}\left(R^{2} \geq 0.8\right) .{ }^{15-17}$ See Figure 1.

\section{Genotyping and imputation}

For 1701 of the 2400 GIRaFH cases DNA was available for additional genotyping. Genotyping was performed using the $50 \mathrm{~K}$ gene-centric Human CVD BeadChip ${ }^{18}$ and genotypes were called using the BeadStudio software (Illumina, San Diego, CA, USA) and subjected to quality control filters at the sample and the SNP level. After genotyping, PLINK v1.07 (http:// pngu.mgh.harvard.edu/purcell/plink/) was used to test the SNPs for population substructure which could introduce false-positive associations. This was done by means of multidimensional scaling implementation. ${ }^{19}$ In addition, the SNPs were subjected to additional quality control filters based on sample size and minor allele frequencies (MAF). Samples with a call rate of $<95 \%$ were excluded from further analysis. Genetic markers with a MAF $<1 \%$ were excluded from further analysis. An identity-by-state analysis was performed to ensure that only Caucasian individuals were included in the final association analyses.

\section{Statistical analysis}

With an effective sample size of 1701 cases and 483 events, the GIRaFH sample has $80 \%$ power to identify statistically significant associations for SNPs

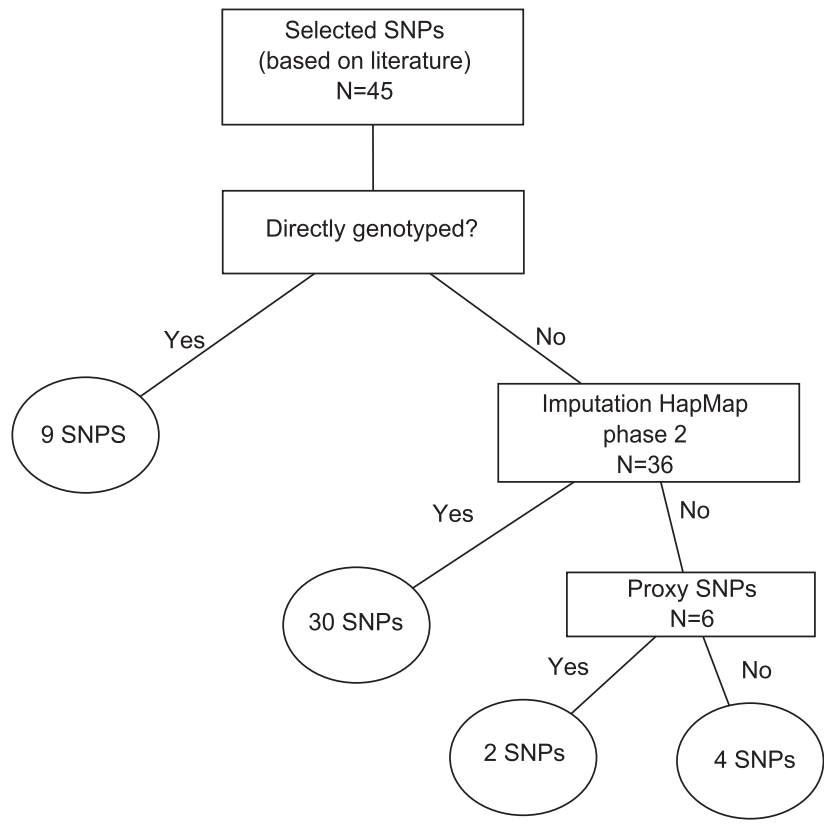

Figure 1 SNP selection procedure. conferring a relative risk $>2.2$ and MAF $>0.10$. Differences between subgroups were tested with $\chi^{2}$ statistics or an independent sample $t$-test where appropriate. Triglycerides had a skewed distribution and therefore statistical analyses were performed on log-transformed data. The association of each SNP with event-free survival time was calculated with a Cox proportional hazard model in the $\mathrm{R}$ package ProbABEL ${ }^{20}$ The event-free survival time was defined as time from birth to date of CAD event, or when no event had occurred as time from birth to date of inclusion in the study. An additive genetic model was applied in the Cox model and classical cardiovascular risks were used as covariates. ${ }^{21}$ We corrected for factors that had previously been shown to be associated with CAD risk in this population: age, gender, smoking, type 2 diabetes, hypertension and body mass index (BMI). Analyses were performed for the loci previously reported to be associated with CAD. Of the 45 reported SNPs, data were available of 41 SNPs after imputation. Significance was defined as a $P$-value $<0.05$ divided by the number of SNPs tested, yielding a significance level of $1.22 \times 10^{-3}$ (41 SNPs based on the literature). All analyses were also performed separately for males and females. Statistical analyses were performed using SPSS software (version 17; Chicago, IL, USA).

All described variants will be submitted to the following public variant database Leiden Open Variation Database (LOVD) ${ }^{3}$ (http://databases.lovd.nl/ shared/genes/).

\section{RESULTS}

\section{Genotyping and imputation}

Of the 1701 DNA samples, 7 individuals did not cluster appropriately in the IBS, reflecting non-Caucasoid origin, and were consecutively excluded, leaving a total of 1694 DNA samples for analysis. A total of 38978 SNPs met our quality control steps. No subjects were excluded because of low call rate. The genomic inflation factor was close to $1(\lambda=1.07)$, indicating that the influence of population substructure and genotyping errors was negligible. Using HapMap, we were able to impute up to 2.5 million SNPs for all individuals. Out of the 45 SNPs, 9 were directly genotyped and 30 were imputed. A proxy of 2 of the remaining 6 SNPs $\left(\mathrm{rs} 12205331: \mathrm{C}>\mathrm{T} \rightarrow \mathrm{rs} 12197124: \mathrm{C}>\mathrm{T}\left(R^{2}=1.0\right)\right.$

\section{Table 1 Baseline characteristics}

\begin{tabular}{|c|c|c|c|}
\hline & $C A D+$ & $C A D-$ & \\
\hline & $\mathrm{n}=482$ & $\mathrm{n}=1212$ & $\mathrm{P}$-value \\
\hline Age first visit lipid clinic (years) & $50.5(11.1)$ & $42.2(12.3)$ & $<0.0001$ \\
\hline Age last visit lipid clinic (years) & $57.1(11.2)$ & $46.6(12.5)$ & $<0.0001$ \\
\hline Male gender & $301(62)$ & $516(42)$ & $<0.0001$ \\
\hline Diabetes mellitus & $54(11)$ & $45(4)$ & $<0.0001$ \\
\hline Hypertension & $79(16)$ & $77(6)$ & $<0.0001$ \\
\hline History of smoking & $365(75)$ & $766(63)$ & $<0.0001$ \\
\hline Body mass index, $\mathrm{kg} / \mathrm{m}^{2}$ & $25.7 \pm 3.2$ & $24.9 \pm 3.6$ & $<0.0001$ \\
\hline Family history CVD & $204(42)$ & $607(50)$ & 0.005 \\
\hline \multicolumn{4}{|l|}{ Treatment-naive lipids levels: } \\
\hline Total cholesterol, mmol/l & $9.6(2.1)$ & $9.4(1.9)$ & 0.146 \\
\hline LDL-cholesterol, mmol/l & $7.0(1.9)$ & $7.2(1.8)$ & 0.152 \\
\hline HDL-cholesterol, mmol/l & $1.1(0.3)$ & $1.2(0.4)$ & $<0.0001$ \\
\hline Triglycerides ${ }^{\mathrm{a}}, \mathrm{mmol} / \mathrm{l}$ & $1.8(1.3-2.5)$ & $1.5(1.0-2.0)$ & $<0.0001$ \\
\hline LDL-receptor mutation proven ${ }^{b}$ & $207(43)$ & $655(54)$ & 0.017 \\
\hline Age of CAD onset, years & $49.1 \pm 10.7$ & $\mathrm{Na}$ & $\mathrm{Na}$ \\
\hline Age of start statin use & $48.8 \pm 11.5$ & $40.9 \pm 12.6$ & $<0.0001$ \\
\hline
\end{tabular}

Abbreviations: CAD, coronary artery disease; CVD, cardiovascular disease; LDL, low-density lipoprotein; HDL, high-density lipoprotein.

Values are given as number (percentage) or mean \pm SD unless indicated otherwise.

alues are given as number

aMean \pm interquartile range.
bMutations of the LDL receptor that have been examined according to Umans-Eckenhausen MA et al. ${ }^{13}$ 


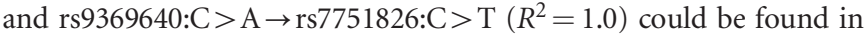
LD with the lead SNP. So a total of 9 SNPs was genotyped directly, 30 SNPs were imputed, for two SNPs, proxy SNPs were found and 4 SNPs could not be found within the imputed data, because they were not available in the reference panel or they were poorly imputed. We only analyzed the 41 SNPs that were available after imputation.

\section{Demographic data}

Demographic data of the 1694 study cases are listed in Table 1. The average age at inclusion was higher in the CAD group. The mean age of onset of CAD was 49.1 (standard deviation; SD 10.7) years and the mean event-free survival in individuals without CAD was 47.3 (SD 12.6) years. During follow-up, $28 \%$ of our cohort developed $\mathrm{CAD}$. Cardiovascular risk factors were significantly more prevalent in
CAD cases than in controls. Treatment-naive LDL-C levels at the time of inclusion in the cohort did not differ between patients with and without CAD. All first visits to the lipid clinic took place between March 1969 and November 2002

\section{SNPs and risk of CAD}

None of the 41 SNPs reached a significant $P$-value after the Bonferroni correction $\left(P<1.11 \times 10^{-3}\right)$ (see Table 2$)$. The best performing SNP in the cardiovascular risk factors adjusted analysis was rs1122608:G>T, in the SMARCA4 gene near the LDLR gene, with HR 0.74 (CI $0.49-0.99$ ) and $P$-value 0.021 (Figure 2). No differences were observed in gender specific analysis (data not shown).

Table 2 The effect of the 41 SNPs analyzed in our study on CAD in FH

\begin{tabular}{|c|c|c|c|c|c|c|c|c|c|c|c|}
\hline SNP & proxy & Chr & Pos & $R A$ & $R A F$ & $H R$ & $\begin{array}{c}95 \% \\
\text { Cl lower }\end{array}$ & $\begin{array}{c}95 \% \\
\text { Cl upper }\end{array}$ & P-value & Nearest gene & $\begin{array}{c}\text { Genotyped/ } \\
\text { imputed }\end{array}$ \\
\hline rs17464857 & & 1 & 220829332 & G & 0.11 & 1.0339 & 0.57 & 1.50 & 0.89 & MIA3 & Imputed \\
\hline rs17114036 & & 1 & 56735409 & G & 0.11 & 1.4222 & 0.98 & 1.86 & 0.11 & PPAP2B & Imputed \\
\hline rs11206510 & & 1 & 55268627 & C & 0.17 & 1.0886 & 0.89 & 1.28 & 0.39 & PCSK9 & Genotyped \\
\hline rs602633 & & 1 & 109623034 & $\mathrm{~T}$ & 0.19 & 1.0458 & 0.85 & 1.24 & 0.66 & SORT1 & Genotyped \\
\hline rs4845625 & & 1 & 152688691 & $\mathrm{~T}$ & 0.44 & 1.0139 & 0.86 & 1.17 & 0.86 & IL6R & Genotyped \\
\hline rs6725887 & & 2 & 203454130 & $\mathrm{~T}$ & 0.14 & 1.2882 & 0.02 & 2.56 & 0.70 & WDR12 & Imputed \\
\hline rs515135 & & 2 & 21139562 & $\mathrm{~T}$ & 0.15 & 1.0793 & 0.86 & 1.30 & 0.50 & APOB & Imputed \\
\hline rs6544713 & & 2 & 43927385 & $\mathrm{~T}$ & 0.33 & 1.0643 & 0.90 & 1.22 & 0.45 & ABCG5-ABCG8 & Imputed \\
\hline rs1561198 & & 2 & 85663500 & C & 0.46 & 0.9765 & 0.82 & 1.13 & 0.77 & VAMP5-VAMP8-GGCX & Imputed \\
\hline rs2252641 & & 2 & 145517931 & $\mathrm{~T}$ & 0.48 & 1.5015 & 1.03 & 1.97 & 0.10 & ZEB2-AC074093.1 & Imputed \\
\hline rs9818870 & & 3 & 139604812 & $\mathrm{~T}$ & 0.16 & 0.2871 & -1.55 & 2.13 & 0.18 & MRAS & Imputed \\
\hline rs1878406 & & 4 & 148613114 & $\mathrm{~T}$ & 0.15 & 0.8840 & 0.66 & 1.11 & 0.29 & EDNRA & Imputed \\
\hline rs7692387 & & 4 & 156854759 & $A$ & 0.2 & 0.0230 & -13.48 & 13.53 & 0.59 & GUCY1A3 & Imputed \\
\hline rs273909 & & 5 & 131695252 & G & 0.15 & 0.8902 & 0.63 & 1.15 & 0.39 & SLC22A4-SLC22A5 & Imputed \\
\hline rs12205331 & rs12197124 & 6 & 35029419 & $\mathrm{~T}$ & 0.21 & 0.6550 & 0.10 & 1.21 & 0.13 & ANKS1A & Imputed \\
\hline rs10947789 & & 6 & 39282900 & C & 0.22 & 0.0001 & -13.46 & 13.46 & 0.19 & KCNK5 & Imputed \\
\hline rs4252120 & & 6 & 161063598 & C & 0.3 & 0.9838 & 0.83 & 1.14 & 0.84 & PLG & Imputed \\
\hline rs9369640 & rs7751826 & 6 & 12900977 & C & 0.37 & 0.9236 & -3.59 & 5.44 & 0.97 & PHACTR1 & Imputed \\
\hline rs2048327 & & 6 & 160783522 & $\mathrm{~T}$ & 0.37 & 0.8940 & 0.73 & 1.06 & 0.18 & SLC22A3-LPAL2-LPA & Imputed \\
\hline rs12190287 & & 6 & 134256218 & C & 0.38 & 1.0986 & 0.73 & 1.46 & 0.61 & TCF21 & Imputed \\
\hline rs2023938 & & 7 & 19003300 & C & 0.12 & 1.1024 & 0.37 & 1.83 & 0.79 & HDAC9 & Imputed \\
\hline rs11556924 & & 7 & 129450732 & C & 0.4 & 0.9822 & 0.83 & 1.14 & 0.82 & ZC3HC1 & Genotyped \\
\hline rs264 & & 8 & 19857460 & G & 0.14 & 0.9500 & 0.72 & 1.18 & 0.66 & LPL & Genotyped \\
\hline rs2954029 & & 8 & 126560154 & $\mathrm{~T}$ & 0.45 & 0.9869 & 0.84 & 1.14 & 0.86 & TRIB1 & Genotyped \\
\hline rs579459 & & 9 & 135143989 & C & 0.25 & 0.9838 & 0.81 & 1.16 & 0.85 & $\mathrm{ABO}$ & Imputed \\
\hline rs3217992 & & 9 & 21993223 & C & 0.37 & 1.0781 & 0.93 & 1.23 & 0.33 & CDKN2BAS1 & Imputed \\
\hline rs12413409 & & 10 & 104709086 & A & 0.07 & 1.0449 & 0.60 & 1.49 & 0.85 & CYP17A1-CNNM2-NT5C2 & Imputed \\
\hline rs2047009 & & 10 & 43859919 & G & 0.44 & 0.9702 & -1.63 & 3.57 & 0.98 & CXCL12 & Imputed \\
\hline rs11203042 & & 10 & 90979089 & $\mathrm{~T}$ & 0.44 & 0.9130 & 0.76 & 1.06 & 0.24 & LIPA & Genotyped \\
\hline rs9326246 & & 11 & 116116943 & C & 0.08 & 0.9456 & 0.64 & 1.25 & 0.72 & ZNF259-APOA5-APOA1 & Imputed \\
\hline rs974819 & & 11 & 103165777 & $\mathrm{~T}$ & 0.18 & 0.4280 & -0.58 & 1.44 & 0.11 & PDGFD & Imputed \\
\hline rs3184504 & & 12 & 110368991 & C & 0.44 & 0.9943 & 0.84 & 1.15 & 0.94 & $\mathrm{SH} 2 \mathrm{~B} 3$ & Genotyped \\
\hline rs9515203 & & 13 & 109847624 & C & 0.31 & 2.8507 & 0.67 & 5.03 & 0.34 & COL4A1-COL4A2 & Imputed \\
\hline rs9319428 & & 13 & 27871621 & G & 0.33 & 1.0639 & 0.90 & 1.22 & 0.45 & FLT1 & Imputed \\
\hline rs7173743 & & 15 & 76928839 & $\mathrm{~T}$ & 0.45 & 0.9068 & 0.60 & 1.21 & 0.53 & ADAMTS7 & Imputed \\
\hline rs17514846 & & 15 & 89217554 & C & 0.46 & 0.9977 & 0.85 & 1.15 & 0.98 & FURIN-FES & Genotyped \\
\hline rs2281727 & & 17 & 2064695 & G & 0.32 & 1.0620 & 0.80 & 1.32 & 0.65 & SMG6 & Imputed \\
\hline rs12936587 & & 17 & 17484447 & A & 0.47 & 1.0324 & 0.86 & 1.20 & 0.72 & RAI1-PEMT-RASD1 & Imputed \\
\hline rs15563 & & 17 & 44360192 & G & 0.48 & 0.9946 & 0.85 & 1.14 & 0.94 & UBE2Z & Imputed \\
\hline rs1122608 & & 19 & 11024601 & $\mathrm{~T}$ & 0.21 & 0.7415 & 0.49 & 0.99 & 0.02 & LDLR & Imputed \\
\hline rs9982601 & & 21 & 34520998 & C & 0.21 & 0.6858 & -1.43 & 2.80 & 0.73 & Gene desert (KCNE2) & Imputed \\
\hline
\end{tabular}

Abbreviations: CAD, coronary artery disease; FH, familial hypercholesterolemia; SNPs, single-nucleotide polymorphisms. 


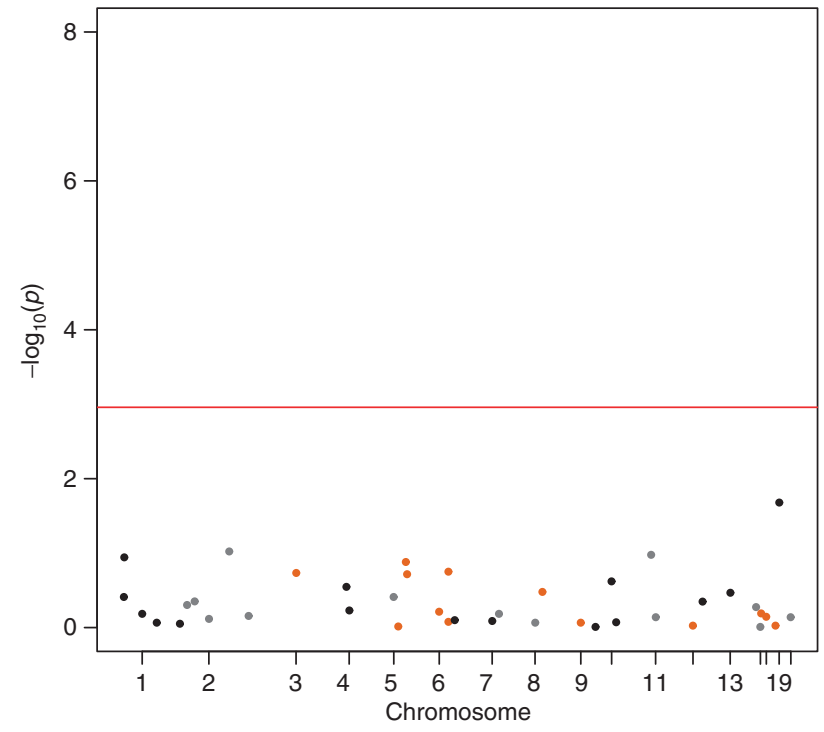

Figure 2 Manhattan plot containing the entire set of analyzed SNPs (41) associated with CAD. The Manhattan plot presents the $-\log _{10}$ ( $P$-value) of all the SNPs analyzed in the current analysis adjusted for cardiovascular risk factors (age, gender, smoking, type 2 diabetes, hypertension and $\mathrm{BMI}$ ). Four of the 45 SNPs associated with CAD were not available for analysis in our data after imputation. The red line represents the $P$-value indicating statistical significance, taking into account the Bonferroni correction for multiple testing.

\section{DISCUSSION}

We tested the hypothesis that common genetic variants that were previously shown in GWA studies to be associated with CAD risk in the general population, might affect the risk of CAD in a high-risk cohort of FH patients. As previously reported, established risk factors do associate with the risk of CAD in FH patients. ${ }^{8,22}$ However, none of the tested CAD-associated SNPs significantly modified the risk of CAD in our FH cohort in analyses unadjusted or adjusted for established cardiovascular risk factors. The lowest observed $P$-value of association was for a SNP in the SMARCA44 gene, near the $L D L R$ gene (in adjusted analysis; $P=0.021$ ); however, it showed a paradoxically protective effect.

FH patients are known to be at high CAD risk. Other patient cohorts with high risk are those with established cardiovascular disease and those with type 2 diabetes. Of these three patient categories, the effect of $9 \mathrm{p} 21$ variants on survival had been tested only in those with established CAD. In a prospective observational study including 846 Caucasoid cases who underwent CABG, the 9p21 SNP rs10116277:G > T was independently associated with all-cause mortality during 5 years follow-up after surgery. ${ }^{23}$ Homozygotes for the minor allele of this SNP had an increased risk of all-cause mortality (HR 1.7; CI $1.1-2.7$ ). The SNP even remained associated with outcome after adjustment for the Euroscore, a score commonly used to predict CVD outcome after CABG. In contrast, in a larger cohort of patients with established CAD ( $>8000$ patients), a haplotype block with eight of the strongest 9p21 SNPs was associated with better prognosis in whites but not in blacks or Hispanics. ${ }^{24}$ Moreover, in line with our findings, the HRs for prognosis among the risk alleles were in opposite direction compared with the published $\mathrm{HRs}$ for CAD/MI risk in the Caucasoid population for both the two most widely reported 9p21 SNPs; rs2383207:A $>\mathrm{G}$ and rs10757278:A $>\mathrm{G}, \mathrm{G}$ alleles were $0.75(0.60-0.93)$ and $P=0.0083$ and $0.81(0.66-1.0) \quad P=0.05$.
However, a less commonly reported linkage disequilibrium consisting of six 9p21 SNPs was associated with worse prognosis. ${ }^{24}$ Compared with these two studies in high-risk populations, our study adds a considerable number of events in a high-risk populations; a total of $482 \mathrm{CAD}$ events occurred during follow-up in our cohort, whereas the studies by Muehlschlegel et a ${ }^{23}$ and Gong et a ${ }^{24}$ reported analyses on 38 and $134 \mathrm{CVD}$ events, respectively. In summary, the only two other studies that addressed the effect of 9p21 SNPs showed conflicting results in high-risk populations.

Previous work conducted by our group to determine the genetic modifiers of CVD risk among FH patients showed that the G20210A polymorphism in the protrombin gene was strongly associated with significantly increased CVD risk. ${ }^{14}$ However, in that publication, the threshold to reach statistical significance was rather lenient. In this paper, a $P$-value $<0.001$ was considered statistically significant; however, applying the Bonferroni correction, which is common practice nowadays would suggest a $P$-value $<0.00076$. None of the SNPs reached the a priori determined value for statistical significance. Because the reported protrombin variant is not considered to be a CAD risk SNP its effect on survival was not calculated in our current analysis. In current analysis, only 1701 samples of the original 1940 were available. A selection bias has not taken place. This is merely a reflection of the usage of the DNA. Baseline characteristic are similar in both studies.

Our study did not address the underlying explanation for the rather counterintuitive result. The top SNP in our analysis, rs1122608:G > T, had a protective effect, contrary to the latest papers. However, smaller reports have reported a protective effect of this variant for CAD and PAD. ${ }^{25,26}$ Martinelli et a ${ }^{25}$ suggest that the effect they observed is due to the lipid effects of the variants. ${ }^{25,26}$

The effect of common CAD-associated genetic variants on general population samples has also yielded conflicting results. In a study among 3000 cases and 3000 controls, a gene score comprising nine SNPs was associated with CAD risk. ${ }^{27}$ People in the top quintile for this gene score had a twofold increased risk of MI compared with those in the bottom quintile corrected for age, sex and ancestry. In line, SNP-based risk score designed in a Finnish cohort of 30725 participants free of CVD was associated with the risk of a first CAD event, with a relative risk of 1.7 between the highest and lowest quintiles of the score adjusted for traditional risk factors; sex, LDL-C, HDL-C, current smoking, BMI, systolic and diastolic blood pressure, blood pressure treatment an prevalent type 2 diabetes. ${ }^{28}$ In contrast, Paynter et $a l^{29}$ did not observe an independent association between genetic risk factors and CAD risk in a cohort of 19313 initially healthy women during 12.3 years follow-up. A risk score based on 12 SNPs was clearly associated with CAD risk after adjustment for age. However, after additional adjustment for other traditional risk factors this association disappeared.

We have previously shown that the type of LDLR mutations underlying $\mathrm{FH}$, variation in LDL-C levels, and established classical risk factors explain $21.3 \%$ of the variation in $\mathrm{CAD}$ risk. Our current analysis suggests that environmental and/or unknown genetic factors may have a role. As there was no standardized information available on lifestyle factors such as dietary habits and physical activity, the effect of environmental factors and their potential interaction with genetic variants could not be studied, but it is unlikely that this could explain significant proportions of the remaining $80 \%$ of CAD risk prediction. At maximum, the common genetic variants we tested explained only $10 \%$ of the heritability of the trait, if we consider the heritability estimates of $40 \%$ for CAD to be correct. ${ }^{10}$ Much of the 'missing heritability' is expected to be explained by common variants 
not yet identified, rare variants, structural variants and copy number variations.

Several aspects of the design of our study need to be considered. The major strength of this study is the unparalleled cohort size, the detailed information on $\mathrm{CAD}$ events during follow-up and the high CAD event rate. However, our study is hampered by several limitations. First, power calculations suggest that our study was at the limit of the power needed to detect statistically significant associations with CAD, as the studied SNPs were previously shown to have a moderate-effect (RR 1.1-1.7). In addition, the MAF of some of the SNPs were lower then used in the power calculation. Second, the majority of the CAD SNPs analyzed were not directly genotyped but imputed using HapMap and we cannot rule out misclassifications. However, Southam et al ${ }^{17}$ have shown that imputation of common variants is generally very accurate. Finally, the patients who were included in this study were referred to a Lipid Clinic. In theory, patients with the most detrimental genetic profiles might have died before referral. Therefore, the effect of genetic variants associated with a more severe $\mathrm{CAD}$ phenotype or early death could have been underestimated or missed.

\section{CONCLUSION}

In this high-risk cohort of patients with $\mathrm{FH}$, common SNPs shown to be associated with CAD risk in the general population could not be associated with the disease.

\section{CONFLICT OF INTEREST}

The authors declare no conflict of interest.

\section{ACKNOWLEDGEMENTS}

This work was supported by Bloodomics consortium, European Union 6th Framework Programme (LSHM-CT-2004-503485), Ipse Movet, the Lifetime Achievement Award of the Dutch Heart Foundation (2010T082) to JJPK and grants from the British Heart Foundation and the National Institute for Health Research, England to WHO. The funders had no role in study design, data collection and analysis, decision to publish or preparation of the manuscript.

1 Goldstein JL, Sobhani MK, Faust JR et al: Heterozygous familial hypercholesterolemia: failure of normal allele to compensate for mutant allele at a regulated genetic locus. Cell 1976; 9: 195-203.

2 Huijgen R, Vissers MN, Defesche JC et al: Familial hypercholesterolemia: current treatment and advances in management. Expert Rev Cardiovasc Ther 2008; 6: 567-581.

3 Van Aalst-Cohen ES, Jansen AC, Tanck MW et al: Diagnosing familial hypercholesterolaemia: the relevance of genetic testing. Eur Heart J 2006; 27: 2240-2246.

4 Stone NJ, Levy RI, Fredrickson DS et al: Coronary artery disease in 116 kindred with familial type II hyperlipoproteinemia. Circulation 1974; 49: 476-488.

5 Sijbrands EJ, Westendorp RG, Defesche JC et al: Mortality over two centuries in large pedigree with familial hypercholesterolaemia: family tree mortality study. BMJ 2001; 322: 1019-1023.
6 Jansen AC, van Aalst-Cohen ES, Tanck MW et al: The contribution of classical risk factors to cardiovascular disease in familial hypercholesterolaemia: data in 2400 patients. J Intern Med 2004; 256: 482-490.

7 Souverein OW, Defesche JC, Zwinderman AH et al: Influence of LDL-receptor mutation type on age at first cardiovascular event in patients with familial hypercholesterolaemia. Eur Heart J 2007; 28: 299-304.

8 Jansen AC, van Aalst-Cohen ES, Tanck MW et al: The contribution of classical risk factors to cardiovascular disease in familial hypercholesterolaemia: data in 2400 patients. J Intern Med 2004; 256: 482-490.

9 Peden JF, Hopewell JC, Saleheen D et al: A genome-wide association study in Europeans and South Asians identifies five new loci for coronary artery disease. Nat Genet 2011; 43: 339-344.

10 Schunkert H, Konig IR, Kathiresan S et al: Large-scale association analysis identifies 13 new susceptibility loci for coronary artery disease. Nat Genet 2011; 43: 333-338.

11 Sivapalaratnam S, Motazacker MM, Maiwald S et al: Genome-wide association studies in atherosclerosis. Curr Atheroscler Rep 2011; 13: 225-232.

12 Deloukas $\mathrm{P}$, Kanoni S, Willenborg $\mathrm{C}$ et al: Large-scale association analysis identifies new risk loci for coronary artery disease. Nat Genet 2012; 45: 25-33.

13 Umans-Eckenhausen MA, Defesche JC, Sijbrands EJ et al: Review of first 5 years of screening for familial hypercholesterolaemia in the Netherlands. Lancet 2001; 357 . 165-168.

14 Jansen AC, van Aalst-Cohen ES, Tanck MW et al: Genetic determinants of cardiovascular disease risk in familial hypercholesterolemia. Arterioscler Thromb Vasc Biol 2005; 25: 1475-1481.

15 Hao K, Chudin E, McElwee J et al: Accuracy of genome-wide imputation of untyped markers and impacts on statistical power for association studies. BMC Genet 2009; 10: 27.

$16 \mathrm{Li} \mathrm{Y,} \mathrm{Willer} \mathrm{CJ,} \mathrm{Ding} \mathrm{J} \mathrm{et} \mathrm{al:} \mathrm{MaCH}$ : using sequence and genotype data to estimate haplotypes and unobserved genotypes. Genet Epidemiol 2010; 34: 816-834.

17 Southam L, Panoutsopoulou K, Rayner NW et al: The effect of genome-wide association scan quality control on imputation outcome for common variants. Eur J Hum Genet 2011; 19: 610-614.

18 Keating BJ, Tischfield S, Murray SS et al: Concept, design and implementation of a cardiovascular gene-centric $50 \mathrm{k}$ SNP array for large-scale genomic association studies. PLoS One 2008; 3: e3583.

19 Purcell S, Neale B, Todd-Brown K et al: PLINK: a tool set for whole-genome association and population-based linkage analyses. Am J Hum Genet 2007; 81: 559-575.

20 Aulchenko YS, Struchalin MV, van Duijn CM: ProbABEL package for genome-wide association analysis of imputed data. BMC Bioinformatics 2010; 11: 134.

21 Jansen AC, van Aalst-Cohen ES, Tanck MW et al: Genetic determinants of cardiovascular disease risk in familial hypercholesterolemia. Arterioscler Thromb Vasc Biol 2005; 25: 1475-1481.

22 Khot UN, Khot MB, Bajzer CT et al: Prevalence of conventional risk factors in patients with coronary heart disease. JAMA 2003; 290: 898-904.

23 Muehlschlegel JD, Liu KY, Perry TE et al: Chromosome 9p21 variant predicts mortality after coronary artery bypass graft surgery. Circulation 2010; 122: S60-S65.

24 Gong Y, Beitelshees AL, Cooper-Dehoff RM et al: Chromosome 9p21 haplotypes and prognosis in white and black patients with coronary artery disease. Circ Cardiovasc Genet 2011; 4: 169-178.

25 Martinelli N, Girelli D, Lunghi B et al: Polymorphisms at LDLR locus may be associated with coronary artery disease through modulation of coagulation factor VIII activity and independently from lipid profile. Blood 2010; 116: 5688-5697.

26 Murabito JM, White CC, Kavousi M et al: Association between chromosome 9p21 variants and the ankle-brachial index identified by a meta-analysis of 21 genome-wide association studies. Circ Cardiovasc Genet 2012; 5: 100-112.

27 Kathiresan S, Voight BF, Purcell S et al: Genome-wide association of early-onset myocardial infarction with single nucleotide polymorphisms and copy number variants. Nat Genet 2009; 41: 334-341.

28 Ripatti S, Tikkanen $\mathrm{E}$, Orho-Melander $\mathrm{M}$ et al: A multilocus genetic risk score for coronary heart disease: case-control and prospective cohort analyses. Lancet 2010; 376: 1393-1400.

29 Paynter NP, Chasman DI, Buring JE et al: Cardiovascular disease risk prediction with and without knowledge of genetic variation at chromosome 9p21.3. Ann Intern Med 2009; 150: 65-72. 